Document downloaded from:

http://hdl.handle.net/10251/177801

This paper must be cited as:

Zakaria, M.; Antonino Daviu, E.; Ferrando Bataller, M. (2020). Radiation Pattern Agile Antenna using PIN Diodes and SPDT Switches. IEEE. 1771-1772.

https://doi.org/10.1109/IEEECONF35879.2020.9329761

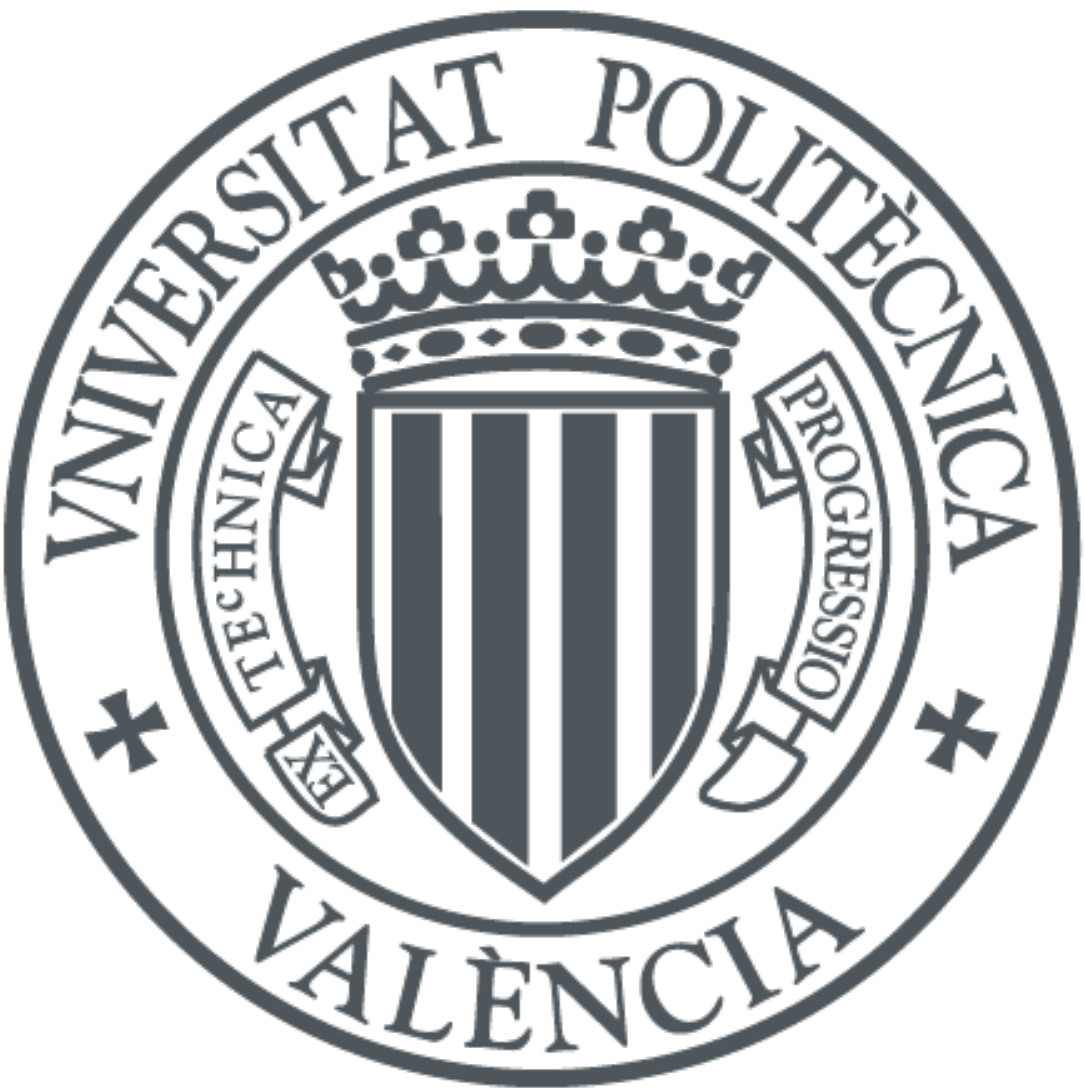

The final publication is available at

https://doi.org/10.1109/IEEECONF35879.2020.9329761

Copyright IEEE

Additional Information 


\title{
Radiation Pattern Agile Antenna using PIN Diodes and SPDT Switches
}

\author{
Zakaria Mahlaoui $^{1,2}$, Eva Antonino-Daviu ${ }^{1}$, Miguel Ferrando-Bataller ${ }^{1}$ \\ ${ }^{1}$ Instituto de Telecomunicaciones y Aplicaciones Multimedia, Universitat Politècnica de València, Spain \\ ${ }^{2}$ National School of Applied Sciences, Cadi Ayyad University, Marrakesh, Morocco \\ mahza@doctor.upv.es
}

\begin{abstract}
In this paper, a new design of a reconfigurable antenna is proposed. The antenna structure is using two PIN diodes for switching between two radiation patterns (directional and bidirectional) and a SPDT switch to achieve good impedance matching. The simulated results show a good impedance matching, with $S_{11}$ below -10 dB using the SPDT switch configuration. In addition, the far-field directivity for both configurations is around $5 \mathrm{~dB}$.
\end{abstract}

Index Terms-Reconfigurable antenna, CMA, Radiation pattern, PIN Diode, SPDT.

\section{INTRODUCTION}

Reconfigurable antennas are required by a significant number of modern wireless communication systems. Their excellent ability to modify some antenna parameter such as radiation pattern [1], polarization [2] and operating frequency [3], makes them a good solution to beat space volume, wireless standard diversity and user-effect problems.

The Characteristic Mode (CM) theory [4], [5] has an enormous potential in antenna engineering, since it delivers an easy approach to physically understand the characteristics of an antenna, such as the radiation pattern, polarization and bandwidth. In many designs [3]-[6], the CM theory has proved its great potential and insight toward physical behavior understanding, what leads to devise antenna systems with high performance.

This work is an improvement of the previous design presented in [1]. Based on the Characteristic Mode Analysis (CMA) shown in the previous work, two PIN diodes were used in two parallel plates to switch between two radiation pattern directions. In the earlier design, the antenna port was located at the the direction of the radiation pattern. In the current design, the antenna port was located at the edge of the plates, far away from the direction of the radiation pattern maximum. A SPDT (Single Pole Double Throw) [7] switch is inserted in order to perform the adequate impedance matching. One of the two outputs of the SPDT is connected directly to the antenna to obtain a bidirectional radiation pattern (case 1), whereas a circuit matching located between the second output of the SPDT and the antenna is adjusted to produce a directional radiation pattern (case 2).

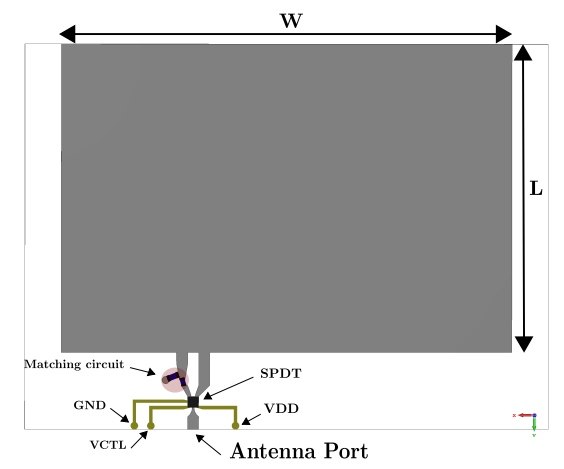

(a)

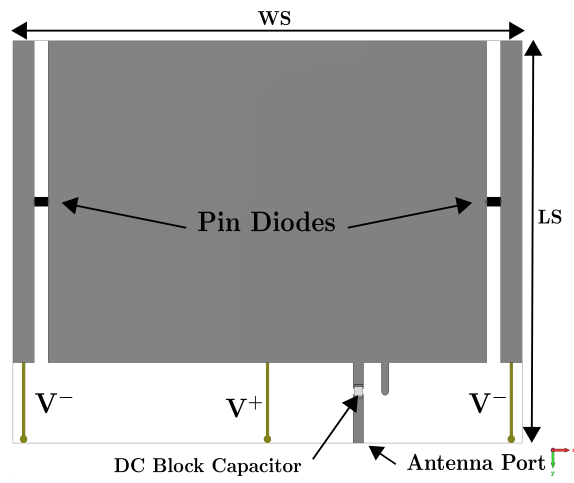

(b)

Fig. 1. (a) Antenna top view: (b) Antenna bottom view.

\section{Proposed antenna Design}

The basic structure was proposed based on a CMA in [1]. It consists in two PEC parallel rectangular plates with the same size. The CMA showed that there are two main modes: Antenna mode $J_{1}$ (where current flows in the same direction in both plates) and transmission line mode $J_{1}^{\prime}$ (where current flows in opposite direction in both plates). However, by exciting the structure using a probe, it is observed that the resulting radiation pattern gets varied from a bidirectional beam to a directional pattern by means of slightly expanding the dimension of one plate.

\section{A. Antenna geometry}

The new proposed structure for the antenna is shown in Fig. 1 and its dimensions are presented in the Table I] The top 
TABLE I

ANTENNA DIMENSION PARAMETERS.

\begin{tabular}{|c|c|c|c|c|c|c|}
\hline Parameter & $\mathrm{W}$ & $\mathrm{L}$ & $\mathrm{WS}$ & $\mathrm{LS}$ & $\mathrm{H}$ & $\epsilon_{r}$ \\
\hline Value (mm) & 28 & 41 & 47.6 & 35 & 1.524 & 2.2 \\
\hline
\end{tabular}

plate of the antenna contains the matching switching network. It consists of the main antenna input port of $50 \Omega$ that is connected to a SPDT in order to choose the appropriate line to feed the antenna, depending on the desired configuration (case 1 or case 2, see Table III). The lower plate is considered as the ground plane, and its dimension can be increased by means of two PIN diodes connected at the two edge arms located at each side, as shown in Fig. 11(b).

\section{B. Bias circuit configuration}

The antenna switches are divided in two roles: the PIN diodes are controlling the switching between the two cases that provide the two different radiation patterns, whereas the SPDT switch is intended to match switching. To perform a good DC biasing supply to the PIN diodes, a DC block capacitor is introduced between the port and the ground plane. To decrease the matching lumped elements in the structure, case 1 is matched by putting the input transmission line at the appropriate position. Case 2 is matched by a series and a shunt capacitors both of them of $0.6 \mathrm{pF}$.

TABLE II

ANTENNA SWITCHES CONFIGURATION.

\begin{tabular}{|c|c|c|c|}
\hline Case & Mode & PIN Diodes & SPDT \\
\hline 1 & $J_{1}^{\prime}$ & OFF & VDD $=5$ V, VCTL $=0 \mathrm{~V}$ \\
\hline 2 & $J_{1}^{\prime}+J_{1}$ & ON & VDD $=5 \mathrm{~V}$, VCTL $=3.3 \mathrm{~V}$ \\
\hline
\end{tabular}

\section{Results and discussions}

After presenting the configuration of the proposed structure, a full-wave simulation is carried out using CST Microwave Studio. All electronic components are taken into consideration in the simulation in order to provide the most precise results. The antenna design and the switches setup are optimized to be controlled with a microcontroller. The antenna radiation pattern structure is based on the two configurations shown in Table 凹;

Case 1: To excite mode $J_{1}^{\prime}$, the PIN diodes are reverse biased and a nought is given to the SPDT $V_{C T L}(0 \mathrm{~V})$. The $S_{11}$ parameter in Fig. 2, with blue line, shows the band obtained at $2.44 \mathrm{GHz}$ with this configuration. As a result, a bidirectional radiation pattern is achieved, with a directivity of $4.8 \mathrm{~dB}$ at both directions $\left(\theta=0^{\circ}\right.$ and $\left.\theta=180^{\circ}\right)$, as presented in Fig. 3(a).

Case 2: The combination of modes $J_{1}^{\prime}$ and $J_{1}$ is excited through the forward bias of the PIN diodes and by altering the $V_{C T L}$ of the SPDT to $3.3 \mathrm{~V}$. This second configuration shows a $S_{11}$ parameter with a bandwidth larger than the first configuration, as shown in Fig. 2. Both configurations go along with the $2.4 \mathrm{GHz}$ band. As a consequence, a directional radiation pattern is obtained with a directivity of $5 \mathrm{~dB}$, as displayed in Fig. 3(b).

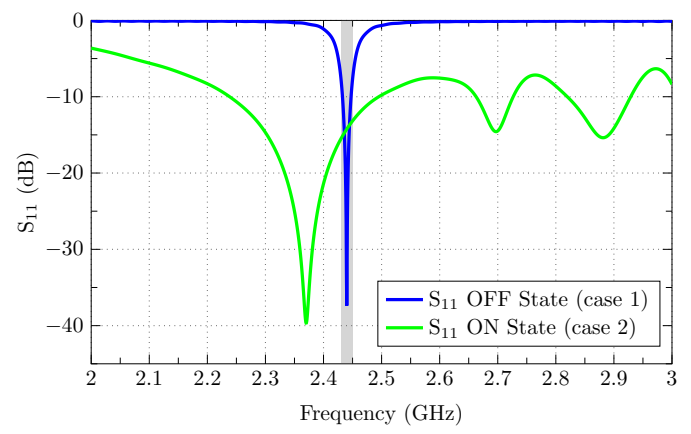

Fig. 2. $S_{11}$ parameters of the case 1 and case 2 .

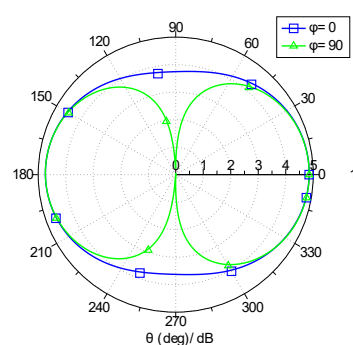

(a)

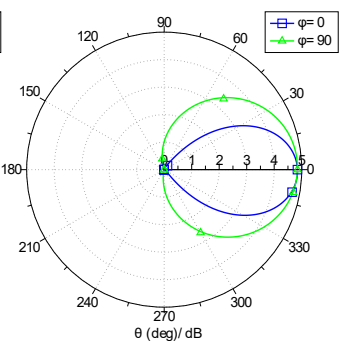

(b)
Fig. 3. Simulated radiation patterns: (a) Case 1; (b) Case 2.

\section{CONCLUSION}

In this design, the issues of the feeding cable and the matching of the design presented earlier in [1] are resolved. A radiation pattern reconfigurable antenna is proposed with a suitable control of the pattern, without drastically changing the antenna structure.

\section{REFERENCES}

[1] Z. Mahlaoui, E. Antonino-Daviu, A. Latif, M. Ferrando-Bataller and C. R. Peńafiel-Ojeda "From the Characteristic Modes Analysis to the Design of a Radiation Pattern Reconfigurable Antenna" The 13th European Conference on Antennas and Propagation (EuCAP) 2019.

[2] A. Bhattacharjee, S.Dwari and M.K Mandal, Polarization-reconfigurable compact monopole antenna with wide effective bandwidth. IEEE Antennas and Wireless Propagation Letters, vol 18(5), pp.1041-1045, 2019.

[3] Z. Mahlaoui, E. Antonino-Daviu, M. Ferrando-Bataller and A. Latif, "Design of a Dual-Band Frequency Reconfigurable Patch Antenna Based on Characteristic Modes," International Journal of Antennas and Propagation, vol 2019, Article ID 4512532, 12 pages, 2019.

[4] P. Futter, "Changing Design with Characteristic Mode Analysis" Microwave journal Altair Engineering Inc, 2016.

[5] R. F. Harrington and J. R. Mautz, "Theory of characteristic modes for conducting bodies," IEEE Transactions on Antennas Propagation, vol. AP-19, no. 5, pp. 622-628, Sept. 1971.

[6] E. Antonino-Daviu, M. Cabedo-Fabres, M. Gallo, M. Ferrando-Bataller and Bozzetti "Design of a Multimode MIMO Antenna Using Characteristic Modes",The 3rd European Conference on Antennas and Propagation (EuCAP) 2009.

[7] Data Sheet "SKY13585-679LF: 1.0 to $6.0 \mathrm{GHz}$ SPDT Switch", Skyworks Solutions, 2015-2017. 